\title{
Abstracts of the meeting of the Clinical Genetics Society held on 7 and 8 April 1983 at the University of Cambridge
}

\begin{abstract}
Clinical application of linked DNA polymorphisms to genotype prediction in $\mathrm{X}$ linked muscular dystrophy

PETER S HARPER

Section of Medical Genetics, Department of Medicine, Welsh National School of Medicine, Cardiff:

Genetic linkage has been shown to exist between the locus for Duchenne muscular dystrophy (DMD) and two DNA restriction fragment length polymorphisms on the short arm of the $\mathrm{X}$ chromosome, each being located at a distance of approximately 15 centiMorgans on either side of the DMD locus. The linkage is not sufficiently close to use for antenatal prediction, but can be used to provide odds for carrier detection. The most accurate prediction is possible in the small number of families informative for both polymorphisms. For the larger number, where only one polymorphism gives information, combination with creatine kinase data may considerably increase the precision of prediction. Examples of families tested will be given and the current limitations of the approach discussed.
\end{abstract}

Clinical use of DNA probes linked to the Duchenne muscular dystrophy (DMD) locus

M E PEMBREY, $K$ E DAVIES, $R$ G Ellis, $R$ WILliamson, T A FAZZONE, C WALKER, AND R M WINTER

Institute of Child Health, London, and Department of Biochemistry, St Mary's Hospital Medical School, London.

The first use of the DMD-DNA probe, $\lambda \mathrm{RC}$, in pregnancy is reported. LS presented at the 12 th week of pregnancy believing (wrongly) that she definitely did not carry the DMD gene. Her mother, an obligatory DMD carrier, was heterozygous for a new, rare, TaqI restriction fragment length polymorphism (RFLP) revealed by the cloned probe $\lambda \mathrm{RC}$, which maps approximately $20 \mathrm{cM}$ from the DMD locus. After family linkage studies the risk of LS being a carrier was reassessed at $76 \%$, in time for her decision about fetal sexing. Of the 70 DMD families on the $\mathrm{ICH}$ register, $18 \%$ include daughters or sisters of obligate carriers. Remaining families have a single affected boy, but in half there is also a normal brother, allowing possible reassessment of the probability of new mutation by linkage analysis. Using $\lambda \mathrm{RC} 8$ and another probe which demonstrates an RFLP, L1.28, $60 \%$ of women should be heterozygous, and all but one of our families could benefit from linkage studies if informative.
Genetic linkage between Becker muscular dystrophy and polymorphic DNA probes on the short arm of the $X$ chromosome

H M KINGSTON AND P S HARPER

Section of Medical Genetics, Department of Medicine, Welsh National School of Medicine, Heath Park, Cardiff.

DNA restriction fragment length polymorphism (RLFP) studies in Becker muscular dystrophy (BMD) have shown eight families informative for the cloned sequence L1.28, which is located on the short arm of the $X$ chromosome between Xp11.0 and Xp11.3. Analysis of these families reveals linkage between these loci, with the maximum likelihood estimate of the genetic distance being 16 centiMorgans $(95 \%$ confidence limits 15 to $16.5 \mathrm{cM})$. These results, together with those suggesting linkage between BMD and $\lambda$ RC8 probe, located between $\mathrm{Xp} 21$ and $\mathrm{Xp} 22 \cdot 3$, indicate a location for the BMD genes on the short arm of the $\mathrm{X}$ chromosome. Similar result obtained in previous studies of Duchenne musculas dystrophy (Murray et al, Davies et al) raise the possibilit that these two disorders may be allelic.

Fetal sex determination using male specific DNA probes and karyotype analysis from chorionic villus biopsies in first trimester pregnancy

J R GOSDEN, C M GOSDEN, C H RODECK, AND J M MORSMAN

MRC Clinical and Population Cytogenetics Unit, Western General Hospital, Crewe Road, Edinburgh, and Department of Obstetrics, King's College Hospital Medical School, Denmark Hill, London SES.

Restriction endonuclease analysis of $\mathrm{Y}$ chromosome specific DNA and fetal karyotype analysis were carried out on chorionic biopsy specimens taken in the first trimester of pregnancy. Chorionic villi were obtained transcervically under direct vision with ultrasound guidance. Twenty-nine specimens have been analysed by restriction endonuclease digestion and hybridisation of Y chromosome specific DNA probes. In every case the result was confirmed by karyotype analysis of cells cultured from a parallel biopsy and from the products of conception. Identification of male fetuses at 8 weeks' gestation gives time for specific investigations to be performed, while still allowing the possibility of early termination if the fetus is affected, and provides rapid reassurance for the parents if the fetus is female. Comparative advantages and disadvantages of cloned and 
uncloned $\mathrm{Y}$ chromosome specific DNA probes will be discussed, together with the problems of culture of cytotrophoblast and mesenchyme and their possible solution.

Intra- or extragenic deletions are associated with EhlersDanlos syndrome type II and broad boned lethal osteogenesis imperfecta

F M POPE, F G GROSVELD, AND A C NICHOLLS

MRC Clinical Research Centre, Watford Road, Harrow, and Laboratory of Gene Structure and Expression, National Institute for Medical Research, Mill Hill, London.

Using a $38 \mathrm{~kb}$ Cosmid insert to an $\alpha \mathrm{l}$ (I)-like collagen gene we have identified a variety of intra- or extragenic deletions in patients with Ehlers-Danlos syndrome type I and II and broad boned lethal osteogenesis imperfecta. We have now identified five patients with broad boned lethal osteogenesis imperfecta who are apparently homozygous for a deletion similar to that which in its heterozygous form causes EDS II. So far we have identified three of four parents of two of those children as being heterozygous for the gene. Antenatal diagnosis of this variant now seems possible provided that adequate DNA for Southern blotting experiments can be provided. The recurrence risk of this variety should now be modified to 1 in 4.

Fc receptor and macrophage lymphocyte interaction (IA): possible human monocyte/macrophage genetic markers indicating susceptibility to leprosy

N F MISTRY, T J BIRDI, P R MAHADEVAN, AND N A ANTIA

Foundation for Medical Research, Worli, Bombay, India.

Repeated studies in long term treated bacteriologically negative lepromatous patients revealed two basic defects in their macrophage function: a reduction in the ability to express Fc receptor on the plasma membrane and the inability to interact with lymphocytes (IA). Both these features were expressed on in vitro challenge of these macrophages with viable Mycobacterium leprae. These same parameters were studied in contacts of lepromatous patients along with the in vivo Mitsuda lepromin reaction with the object of identifying putative genes that may code for susceptibility to lepromatous leprosy. The 20 families studied consisted of either parent being the index case. The normal partner and the progeny were monitored as contacts (total 85). Except for two families, all the progeny were clinically unaffected. Appropriate normal controls (occupational contacts and the general population of a hyperendemic area) were also sampled. Either one of the in vitro parameters showing a defective response classified the person as susceptible. The susceptible phenotype was expressed in a 3:1 ratio over the normal phenotype in the progeny of the index cases, the Fc and IA patterns segregating independently. Mendelian analysis showed that the susceptible genotype could be inherited in a heterozygous dominant form from the affected parent and expressed as such. The present study defines two parameters (Fc receptor and macrophage-lymphocyte interaction) as genetic markers of susceptibility to leprosy present predominantly on the peripheral blood monocyte/ macrophage that identifies subjects at risk long before the manifestation of overt disease.

A computerised data base for the diagnosis of dysmorphic syndromes

R M WINTER AND M BARAITSER

Kennedy Galton Centre, Harper Lane, Radlett, Herts, and Clinical Genetics Unit, Hospital for Sick Children, Great Ormond Street, London.

Seven in 1000 babies are born with multiple malformations. Many of these children will have a syndrome that has been previously recognised and described, albeit in a single paper. Most malformation syndromes are individually rare but the genetic implications are of extreme importance to families. We describe a system whereby these syndromes can be recalled, using a computerised literature data base running on a microcomputer. The syndromes in the data base have appeared in genetic and paediatric journals in the past 15 years and the data base can be easily updated. The information is entered so that the computer can be asked for all syndromes and references with a certain combination of clinical features, for example, polydactyly and mental retardation. If the list is too long, other clinical features can be added. Alternatively, a completely different combination of clinical features can be entered. Because the retrieval programme is flexible, easy to use, and accessible on a microcomputer, it is already proving to be a valuable working tool for the assessment of patients with dysmorphic features.

Dysmorphology of the Poland anomaly, isolated absence of the pectoralis major, and allied disorders

T J DAVID

Booth Hall Children's Hospital, Charlestown Road, Blackley, Manchester.

Forty-six patients with the Poland anomaly and 32 patients with isolated absence of the pectoralis major muscle were studied. There were 45 males and 33 females, a ratio of $1 \cdot 36$. There were 42 right sided cases and 36 left sided ones, a ratio of $1 \cdot 17$. The excess of right sided cases was confined to females. The clinical features of the Poland anomaly were extremely variable, but there was no correlation between the severity of the thoracic and limb defects in individual patients. The digital abnormalities varied from dermatoglyphic defects only, in the presence of a normal hand, to mild cutaneous syndactyly and brachydactyly, to total absence of all five digits. The thoracic defect varied from partial absence of the sternocostal head of the pectoralis major to a severe chest wall defect with rib cage defects, absence of the breast, and 
absence of other shoulder girdle muscles (serratius anterior, latissimus dorsi). Palmar dermatoglyphs were normal on the unaffected side, confirming the unilaterality of the Poland anomaly.

\section{Genetics in art}

ALAN E H EMERY

The Medical School, Teviot Place, Edinburgh.

Occasionally a genetic disorder has afflicted the painter himself and this has then been reflected in his work, a notable example being Fernand Léger's colour blindness. More interestingly, however, is the actual portrayal in art of subjects with genetic disorders. Examples can be found from earliest times and include various forms of dwarfism in ancient Egyptian art, intersex in Carthaginian art, and dysmorphic features in American Indian art. There are then the portraits of distinguished personages who we now know had various inherited disorders such as the Hapsburg lip in Charles V (Titian) and porphyria in George III (Zoffany). Sometimes the artist in a selfportrait has revealed abnormalities in himself such as strabismus (Dürer) and cyanotic heart disease (Ket). Finally, there are the numerous examples of genetic disorders which have been recorded by observant and careful artists, such as congenital Cushing's syndrome (Carreño), dwarfism (Velasquez), hypertelorism (Goya), epilepsy (Raphael), talipes (Ribera), blindness (Millais), and polydactyly (Chagall). Clearly, art has much to interest the clinical geneticist.

\section{A maximum likelihood estimate of the sex ratio of mutation rates in haemophilia $A$}

R M WINTER, E G D TUDDENHAM, E GOLDMAN, AND K B MATTHEWS

Division of Inherited Metabolic Diseases, Northwick Park Hospital, Harrow; Kennedy Galton Centre, Harperbury Hospital, Radlett; and The Katharine Dormandy Haemophilia Centre and Haemostasis Unit, The Royal Free Hospital, London.

In order to estimate the sex ratio of mutation rates in haemophilia A by the method of Winter ( $\mathrm{Am} \mathrm{J} \mathrm{Hum} \mathrm{Genet}$ $1980 ; 32: 582-8)$, carrier detection tests were carried out on 21 mothers of isolated cases of severe haemophilia A, according to WHO recommendations. A maximum likelihood estimate of the male to female mutation ratio of $9.6(5 \%$ confidence limits $2 \cdot 2$ to $41 \cdot 5)$ was obtained. These results will be discussed in relation to similar studies for other X linked loci.

Acrocephalosyndactyly of non-Apert (I) type: how many different genetic entities?

A SCHINZEL, M WOODTLI, AND U BURCK

Institute of Medical Genetics, University of Zurich, and Institute of Human Genetics, University of Hamburg.

In addition to the well-established Apert syndrome (type I), most classifications of acrocephalosyndactylies include the Saethre-Chotzen syndrome and the Pfeiffer syndrome; recently, a further type (Robinow-Soraf syndrome) has been proposed. We have examined 24 patients of nonApert acrocephalosyndactyly including five sporadic cases and familial cases from four families. Marked variability of clinical features was found in cases within a family. For example, skull index and asymmetry varied considerably from member to member. In some members of one family with eight affected, features of the SaethreChotzen syndrome prevailed, while others showed the Pfeiffer phenotype. In another family, the daughter revealed duplication of metatarsals and phalanges of the big toes (compatible with the Robinow-Soraf syndrome) while her mother had the Pfeiffer syndrome with hypoplastic and angulated thumbs and big toes. One sporadic case had unilateral duplication of big toe phalanges. The intrafamilial variability of clinical features in our series suggests that Saethre-Chotzen, Pfeiffer, and Robinow-Soraf syndromes are not different genetic entities, but rather the result of variable clinical expression of one and the same mutant gene. Thus, there are probably two autosomal dominant types of acrocephalosyndactyly, the Apert type and the (clinically milder) non-Apert type.

A possible $X$ linked form of Leigh's syndrome (infantile subacute necrotising encephalopathy)

T GRIMM, M BRACKERTZ, W GÖRKE, H KOLLASCHINSKI U PFEIFER, AND D SCHINDLER

Humangenetik, Kinderklinik, and Pathologie, UniversitäE Wurzburg, Germany.

The possibility of an $\mathrm{X}$ linked form of Leigh's syndrome was suggested in a recent review. (Benke et al, Hum Genet $1982 ; 62: 52$ ) which documented a raised male/female ratio and a significant excess of affected male sibs. We observed a 3-year-old boy who died with typical clinical signs of Leigh's syndrome corroborated by necropsy brain findings. The parents are unrelated (the father being of German and the mother of Italian ancestry). Pyruvate carboxylase and pyruvate dehydrogenase levels in cultured fibroblasts of the father were normal, whereas they were raised in cells of both mother and affected son. In analogy to other $\mathrm{X}$ linked disorders, this rise may be explained as a secondary metabolic disturbance. As in similar published pedigrees, the possibility of $X$ linked inheritance of the, as yet undetermined, gene defect must be considered.

Genotype prediction and gene mapping in myotonic dystrophy

T O'BRIEN

Section of Medical Genetics, Department of Medicine, Welsh National School of Medicine, Cardiff.

A clinical and genetic linkage study of myotonic dystrophy was undertaken. The clinical conclusions were that the risk to a normal sib in a congenital myotonic sibship is considerably less than the dominant mode of inheritance 
predicts, that hypotension is a prominent feature of myotonic dystrophy, that a raised $\mathrm{LH}$ level may be a more useful early indicator of heterozygosity than a raised FSH level, and that asymptomatic heterozygotes may play a prominent role in gene survival in the community. A genetic linkage study has shown that peptidase $D$ is closely linked to myotonic dystrophy and has confirmed the assignment of the $\mathrm{C} 3$ component of complement to the same linkage group.

\section{Chromosome studies in the Prader-Willi syndrome \\ C N FEAR, D E MUTTON, V DUBOWITZ, AND A C BERRY}

Paediatric Research Unit, Guy's Hospital, London, and Department of Paediatrics and Neonatal Medicine, Hammersmith Hospital, London.

Seventeen children whose clinical features were considered by one observer (VD) to be either typical of the Prader-Willi (PW) syndrome or highly suggestive of it were karyotyped using both good quality routine Giemsa stained preparations and a high resolution technique. Parental karyotypes were examined where available. Preparations from 20 unrelated controls were critically examined and found to be normal. Five Prader-Willi children appeared to be chromosomally normal. One had an unbalanced chromosome complement derived from a familial translocation $(15 ; 22)(q 13 ; q 11.2)$. Six children had a deletion of band 15q12. One child appeared to have a deletion of half of this band but his father had a similar 15 . One child had an unusually long $18 \mathrm{p}$. In two cases the preparations gave inadequate resolution for fine analysis and one result was equivocal. The level of variation in the q12 region of chromosome 15 was considerable, leading to potential difficulty in interpretation of results.

The use of interspecific in vitro fertilisation for the visualisation of the chromosomes of human sperm

P T TOMKINS, C CARROLL, S WALSH, AND J A HOUGHTON

Department of Microbiology, University College, Galway, Ireland.

The only non-invasive and, indeed, ultimately valid method for determining the incidence of structural and numerical cytogenetic aberrations arising among the products of spermatogenesis is to sample directly the sperm chromosomes of a typical ejaculate. Potential methods for visualising sperm chromosomes are: (a) active or passive interspecific in vitro fertilisation; (b) sperm chromosome decondensation within somatic cells following active or passive fusion; and (c) microinjection of sperm nuclei into foster eggs or somatic cells. A modification of the technique of passive interspecific in vitro fertilisation described by Rudak et al (1978) has been used for several months and five major parameters have been found to contribute to the success of this method: (a) quality of the original sample; (b) method of sperm preparation; (c) the fertilisation medium; (d) capacitation conditions; and (e) mechanics and kinetics of gamete interaction. The modifications of the original technique will be described. Methods for the stabilisation and improvement of low fertilisation rates will be reported, and standardisation of capacitation patterns by active sperm protein coat removal and initial chromosome analysis and banding results will be described.

No evidence for a paternal age effect on the frequency of trisomy 21 at amniocentesis in 13300 pregnancies: an analysis of data from a European collaborative study

J R W YATES AND M A FERGUSON-SMITH

Duncan Guthrie Institute of Medical Genetics, University of Glasgow, Yorkhill, Glasgow.

It has been suggested that the well-known rise in the incidence of trisomy 21 with increasing maternal age is complemented by a similar independent paternal age effect (Stene et al, Hum Genet 1981;59:119-24). Thanks to the kind collaboration of 58 centres in the United Kingdom, Europe, Canada, and South Africa, the results of chromosome analysis, together with maternal and paternal age, have been collected for 13300 amniocenteses carried out because the mother's age was greater than 35 years (Ferguson-Smith and Yates, Prenatal Diagnosis, in preparation). Preliminary analysis using stepwise logistic regression shows no evidence for a significant paternal age effect independent of maternal age (improvement in fit $\chi^{2}=0.004, \mathrm{df}=1, \mathrm{p}$ value $=$ $0.95)$. This finding is discussed in the context of previously published studies and their methodology.

Possible use of microvillar peptidase activity in amniotic fluid for the antenatal diagnosis of cystic fibrosis

N J B CARBARNS, C M GOSDEN, D J H BROCK, AND C HAYWARD

$M R C$ Clinical and Population Cytogenetics Unit and University Department of Human Genetics, Western General Hospital, Edinburgh.

Cystic fibrosis is a disease involving secretions of exocrine gland epithelial cells. The surface of epithelial cells is characterised by microvilli. $\gamma$-glutamyl transpeptidase (GGTP) and amniopeptidase M (APM) are enzymes of the microvillar membrane whose activity decreases with advancing gestation, suggesting they are phase specific. The activities of these enzymes were examined in 23 samples of amniotic fluid from pregnancies where the mother had a previous child with cystic fibrosis, 14 of which had normal outcomes and nine of which resulted in infants with cystic fibrosis, and 110 control amniotic fluids. Protease titration with 4-methylumbelliferylguanidinobenzoate (MUGB) did not distinguish between affected and unaffected pregnancies. In contrast, the levels of GGTP and APM in amniotic fluids from CF pregnancies are clearly reduced when compared with those of normal at risk pregnancies. Separation between affected and normal is more easily achieved at earlier gestations, suggesting an early pathological effect, but lack of knowledge of early pathology makes it premature to offer antenatal diagnosis by this method. 
The cause of neural tube defects: some experiments and a hypothesis

\section{J SELLER}

Paediatric Research Unit, The Prince Philip Research Laboratories, Guy's Hospital Medical School, London.

The curly-tail mouse is an animal model of neural tube defects (NTD). A series of experiments were undertaken to investigate in these mice, genetically predisposed to NTD, the effects of various teratogens on the rodent central nervous system. The agents were injected into the pregnant mother during formation of the fetal neural tube. Completely unexpected results were obtained with three of these substances, mitomycin $\mathrm{C}$, hydroxyurea, and 5-fluorouracil, for they significantly reduced the number of fetuses with NTD. The three substances have in common the fact that they are inhibitors of DNA synthesis. A hypothesis is put forward that the underlying mechanism causing NTD could be a basic metabolic defect in DNA synthesis which affects cell replication and results in abnormal morphogenesis of the neuraxis.

\section{Early conceptual loss in women at added risk of bearing abnormal offspring}

E M WILLIAMSON, J F MILLER, AND K LINDSAY

Southampton General Hospital, Southampton.

Human chorionic gonadotrophin is produced by the trophoblast at an early stage of development and measurement of this glycoprotein hormone was used to recognise pregnancy from as early as the 7th day after ovulation. In a previous study of volunteer women wishing to conceive, we had found evidence of trophoblastic activity in $59.6 \%$ of exposed ovulatory cycles, but $62 \%$ of these detected pregnancies terminated before 12 weeks of pregnancy. The present study followed women at added risk of bearing abnormal offspring who were trying to conceive. Those women at added risk of neural tube defect offspring and those who had previously had a child with a chromosome abnormality showed no difference in their early conceptual loss compared with the normal population. Mothers over 35 years of age showed trophoblastic activity in only $30 \%$ of exposed cycles and of these pregnancies $72 \%$ terminated before 12 weeks.

The reaction to termination of pregnancy for genetic reasons

J LLOYD AND K M LAURENCE

Department of Community Medicine, Mid Glamorgan Health Authority, and Welsh National School of Medicine, Cardiff.

This paper is the preliminary report of a study undertaken to demonstrate the reaction to termination of pregnancy for genetic reasons and to examine follow-up services that are available. Women resident in Mid Glamorgan who had experienced a termination of pregnancy between 1977 and 1981, either as a result of positive findings during the alphafetoprotein screening programme for neural tube defects, or abnormal chromosome tests following amniocentesis because of known high risks, or other genetic reasons, were interviewed using a structured interview. Three internal comparison groups were formed from those women who had also experienced a spontaneous abortion, previous stillbirth or neonatal death, and a previous therapeutic abortion. Of 48 women interviewed, $\mathbf{7 7} \%$ demonstrated an acute grief reaction, $45 \%$ remained symptomatic at 6 months after termination, and $20 \%$ required treatment. This compares with no such reaction after spontaneous abortion or therapeutic abortion earlier in pregnancy. Response to termination of a wanted pregnancy is similar to stillbirth reaction, but follow up visits by a midwife or general practitioner were limited to only $16 \%$ of the post-termination group.

Hereditary persistence of alphafetoprotein: a new autosomal dominant trait identified in an antenatal screening programme for spina bifida

M A FERGUSON SMITH, H M MAY, E O'HARE, AND D A AITKEN Duncan Guthrie Institute of Medical Genetics, Yorkhill, Glasgow.

A 38-year-old woman had grossly raised serum alph fetoprotein levels at 21 and 23 weeks' gestation in her fifth pregnancy. Ultrasonography revealed a normât singleton pregnancy at the estimated gestation and amniotic AFP levels were found to be normal $(<1 \mathrm{mg} / \mathrm{l})$. Serial serum AFP results continued to be raised throughout pregnancy and in samples taken after delivery. The patient appeared healthy and no explanation for the persisting raised serum AFP levels was found. Serum samples from three of her four children, including her newborn son, were also found to have raised AFP levels. Subsequently, 70 members of the family have been tested and 21 , including nine males, were found to have high levels of AFP in their serum. The trait, which is inherited as an autosomal dominant with complete penetrance, does not seem to be associated with any clinical disability and other serum proteins appear normal. Close linkage with group specific component and the MNS group appears to be excluded. No other affected family has been discovered to date among over 150000 women tested in the west of Scotland serum AFP screening programme, and it is assumed that the condition is likely to be a particularly rare cause of raised serum AFP during pregnancy. Determination of the molecular basis for the defect is being pursued. 